\title{
On some characterizations of Besov spaces of generalized smoothness
}

\author{
Susana D. Moura*1 \\ ${ }^{1}$ CMUC, Department of Mathematics, University of Coimbra, 3001-454 Coimbra, Portugal
}

Received 29 November 2005, accepted 8 October 2006

Published online 6 June 2007

Key words Function spaces of generalized smoothness, characterizations, approximation, differences MSC (2000) 46E35

Dedicated to Professor Frank-Olme Speck on the occasion of his 60th birthday

We present characterizations of the Besov spaces of generalized smoothness $B_{p, q}^{\sigma, N}\left(\mathbb{R}^{n}\right)$ via approximation and by means of differences.

(c) 2007 WILEY-VCH Verlag GmbH \& Co. KGaA, Weinheim

\section{Introduction}

Function spaces of generalized smoothness have been introduced and considered, in particular since the middle of the seventies of the last century, by several authors from different points of view and different degrees of generality. We refer, for instance, to Goldman [9, 10], Kalyabin [13, 14], Merucci [16], Cobos and Fernandez [4], the survey by Kalyabin and Lizorkin [12], Leopold [15], Edmunds and Triebel [6, 7] and Moura [17, 18].

From the standpoint of a Fourier analytic characterization, the recent work by Farkas and Leopold [8] represents a unified and general approach on this topic. Following their approach, we see that, under certain conditions, the Besov spaces of generalized smoothness can be analogously characterized via approximation and by means of differences.

\section{Preliminaries}

\subsection{General notation}

In this paper we shall adopt the following general notation: $\mathbb{N}$ denotes the set of all natural numbers, $\mathbb{N}_{0}=\mathbb{N} \cup\{0\}$, $\mathbb{R}^{n}, n \in \mathbb{N}$, denotes the $n$-dimensional real euclidean space and $\mathbb{R}=\mathbb{R}^{1}$. We always use the equivalence " $\sim$ " in

$$
a_{k} \sim b_{k} \quad \text { or } \quad \phi(r) \sim \psi(r)
$$

to mean that there are two positive numbers $c_{1}$ and $c_{2}$ such that

$$
c_{1} a_{k} \leq b_{k} \leq c_{2} a_{k} \quad \text { or } \quad c_{1} \phi(x) \leq \psi(x) \leq c_{2} \phi(x)
$$

for all admitted values of the discrete variable $k$ or the continuous variable $x$, where $\left(a_{k}\right)_{k},\left(b_{k}\right)_{k}$ are nonnegative sequences and $\phi, \psi$ are nonnegative functions. If $a \in \mathbb{R}$ then $a_{+}:=\max (a, 0)$. We consider here only function spaces defined on $\mathbb{R}^{n}$; so for convenience we shall usually omit the " $\mathbb{R}^{n}$ " from their notation.

\subsection{Sequences}

In this subsection we explain the class of sequences we shall be interested in and some related basic results.

* e-mail: smpsd@mat.uc.pt, Phone: +351 239791150, Fax: +351 239793069 
A sequence $\gamma=\left(\gamma_{j}\right)_{j \in \mathbb{N}_{0}}$ of positive real numbers is said to be admissible if there exist two positive constants $d_{0}$ and $d_{1}$ such that

$$
d_{0} \gamma_{j} \leq \gamma_{j+1} \leq d_{1} \gamma_{j}, \quad j \in \mathbb{N}_{0}
$$

For an admissible sequence $\gamma=\left(\gamma_{j}\right)_{j \in \mathbb{N}_{0}}$, let

$$
\underline{\gamma}_{j}:=\inf _{k \geq 0} \frac{\gamma_{j+k}}{\gamma_{k}} \quad \text { and } \quad \bar{\gamma}_{j}:=\sup _{k \geq 0} \frac{\gamma_{j+k}}{\gamma_{k}}, \quad j \in \mathbb{N}_{0}
$$

Then clearly $\underline{\gamma}_{j} \gamma_{k} \leq \gamma_{j+k} \leq \gamma_{k} \bar{\gamma}_{j}$ for any $j, k \in \mathbb{N}_{0}$. In particular, $\underline{\gamma}_{1}$ and $\bar{\gamma}_{1}$ are the best possible constants $d_{0}$ and $d_{1}$ in (2.1), respectively. Then, the lower and upper Boyd indices of the sequence $\gamma$ are defined, respectively, by

$$
\underline{s}(\gamma):=\lim _{j \rightarrow \infty} \frac{\log \underline{\underline{\gamma}}_{j}}{j} \text { and } \bar{s}(\gamma):=\lim _{j \rightarrow \infty} \frac{\log \bar{\gamma}_{j}}{j}
$$

The above definition is well-posed: the sequence $\left(\log \bar{\gamma}_{j}\right)_{j \in \mathbb{N}}$ is sub-additive and hence the right-hand side limit in (2.3) exists, it is finite (since $\gamma$ is an admissible sequence) and it coincides with $\inf _{j>0} \log \bar{\gamma}_{j} / j$. The corresponding assertions for the lower counterpart $\underline{s}(\gamma)$ can be read off observing that $\log \underline{\gamma}_{j}=-\log \left(\overline{\gamma^{-1}}\right)_{j}$.

The Boyd index $\bar{s}(\gamma)$ of an admissible sequence $\gamma$ describes the asymptotic behaviour of the $\bar{\gamma}_{j}$ 's and provides more information than simply $\bar{\gamma}_{1}$ and, what is more, is stable under the equivalence of sequences: if $\gamma \sim \tau$, then $\bar{s}(\gamma)=\bar{s}(\tau)$ as one readily verifies. The same applies to the lower counterpart.

Observe also that for each $\varepsilon>0$ there are two positive constants $c_{1}=c_{1}(\varepsilon)$ and $c_{2}=c_{2}(\varepsilon)$ such that

$$
c_{1} 2^{(\underline{s}(\gamma)-\varepsilon) j} \leq \underline{\gamma}_{j} \leq \bar{\gamma}_{j} \leq c_{2} 2^{(\bar{s}(\gamma)+\varepsilon) j}, \quad j \in \mathbb{N}_{0}
$$

Example 2.1 We consider some examples of admissible sequences.

(i) The sequence $\gamma=\left(\gamma_{j}\right)_{j \in \mathbb{N}_{0}}$,

$$
\gamma_{j}=2^{s j}(1+j)^{b}(1+\log (1+j))^{c}
$$

with arbitrary fixed real numbers $s, b$ and $c$ is a standard example of an admissible sequence with

$$
\underline{s}(\gamma)=\bar{s}(\gamma)=s .
$$

(ii) Let $\Phi:(0,1] \rightarrow \mathbb{R}$ be a slowly varying function (or equivalent to a slowly varying one) in the sense of [1]. Then, for each $s \in \mathbb{R}$ the sequence $\gamma=\left(2^{s j} \Phi\left(2^{-j}\right)\right)_{j \in \mathbb{N}_{0}}$ is an admissible sequence. Also here we have $\underline{s}(\gamma)=\bar{s}(\gamma)=s$.

(iii) In view of [2, Proposition 1.9.7], the case $\gamma=\left(2^{s j} \Psi\left(2^{-j}\right)\right)_{j \in \mathbb{N}_{0}}$, where now $\Psi$ is an admissible function in the sense of [6] (i.e., a positive monotone function defined on $(0,1]$ such that $\left.\Psi\left(2^{-2 j}\right) \sim \Psi\left(2^{-j}\right), j \in \mathbb{N}_{0}\right)$, can be regarded as a special case of (ii).

Remark 2.2 The examples above have in common the fact that their upper and lower Boyd indices coincide. However, this is not in general the case. Examples 2.2.6 and 2.2.7 in [8], due to Kalyabin, show that an admissible sequence has not necessarily a fixed main order.

\subsection{Function spaces of generalized smoothness}

Let $N=\left(N_{j}\right)_{j \in \mathbb{N}_{0}}$ be an admissible sequence with $\underline{N}_{1}>1$ (recall (2.2)). In particular $N$ is a so-called strongly increasing sequence (cf. [8, Def. 2.2.1]), which guarantees the existence of a number $k_{0} \in \mathbb{N}_{0}$ such that

$$
N_{k} \geq 2 N_{j} \text { for any } k, j \text { such that } k \geq j+k_{0} .
$$

It should be noted that the sequence $N=\left(N_{j}\right)_{j \in \mathbb{N}_{0}}$ plays the same role as the sequence $\left(2^{j}\right)_{j \in \mathbb{N}_{0}}$ in the classical construction of the spaces $B_{p q}^{s}$ and $F_{p q}^{s}$. This will be clear from the following considerations. 
For a fixed sequence $N=\left(N_{j}\right)_{j \in \mathbb{N}_{0}}$, as above, we define the associated covering $\Omega^{N}=\left(\Omega_{j}^{N}\right)_{j \in \mathbb{N}_{0}}$ of $\mathbb{R}^{n}$ by and

$$
\Omega_{j}^{N}=\left\{\xi \in \mathbb{R}^{n}:|\xi| \leq N_{j+k_{0}}\right\}, \quad j=0, \ldots, k_{0}-1,
$$

$$
\Omega_{j}^{N}=\left\{\xi \in \mathbb{R}^{n}: N_{j-k_{0}} \leq|\xi| \leq N_{j+k_{0}}\right\}, \quad j \geq k_{0},
$$

with $k_{0}$ according to (2.5).

Definition 2.3 For a fixed admissible sequence $N=\left(N_{j}\right)_{j \in \mathbb{N}_{0}}$ with $\underline{N}_{1}>1$, and for the associated covering $\Omega^{N}=\left(\Omega_{j}^{N}\right)_{j \in \mathbb{N}_{0}}$ of $\mathbb{R}^{n}$, a system $\varphi^{N}=\left(\varphi_{j}^{N}\right)_{j \in \mathbb{N}_{0}}$ will be called a (generalized) partition of unity subordinated to $\Omega^{N}$ if:

(i)

$$
\varphi_{j}^{N} \in C_{0}^{\infty} \quad \text { and } \quad \varphi_{j}^{N}(\xi) \geq 0 \quad \text { if } \quad \xi \in \mathbb{R}^{n} \quad \text { for any } j \in \mathbb{N}_{0}
$$

$$
\operatorname{supp} \varphi_{j}^{N} \subset \Omega_{j}^{N} \quad \text { for any } j \in \mathbb{N}_{0}
$$

(iii) for any $\alpha \in \mathbb{N}_{0}^{n}$ there exists a constant $c_{\alpha}>0$ such that for any $j \in \mathbb{N}_{0}$

$$
\left|D^{\alpha} \varphi_{j}^{N}(\xi)\right| \leq c_{\alpha}\left(1+|\xi|^{2}\right)^{-|\alpha| / 2} \text { for any } \xi \in \mathbb{R}^{n}
$$

(iv) there exists a constant $c_{\varphi}>0$ such that

$$
0<\sum_{j=0}^{\infty} \varphi_{j}^{N}(\xi)=c_{\varphi}<\infty \text { for any } \xi \in \mathbb{R}^{n}
$$

Before turning to the definition of the Besov spaces of generalized smoothness let us recall that $\mathcal{S}$ denotes the Schwartz space of all complex-valued rapidly decreasing infinitely differentiable functions on $\mathbb{R}^{n}$ equipped with the usual topology and by $\mathcal{S}^{\prime}$ we denote its topological dual, the space of all tempered distributions on $\mathbb{R}^{n}$. If $f \in \mathcal{S}^{\prime}$, then $\mathcal{F} f$ and $\mathcal{F}^{-1} f$ denote the Fourier and the inverse Fourier transform of $f$, respectively. For $\varphi \in \mathcal{S}$ and $f \in \mathcal{S}^{\prime}$ we will use the notation $\varphi(D) f=\left[\mathcal{F}^{-1}(\varphi \mathcal{F} f)\right]$. Furthermore, if $0<p \leq \infty$ and $0<q \leq \infty$ then $L_{p}$ and $\ell_{q}$ have the standard meaning and, if $\left(f_{j}\right)_{j \in \mathbb{N}_{0}}$ is a sequence of complex-valued Lebesgue measurable functions on $\mathbb{R}^{n}$, then

$$
\left\|\left(f_{j}\right)_{j \in \mathbb{N}_{0}} \mid \ell_{q}\left(L_{p}\right)\right\|=\left(\sum_{j=0}^{\infty}\left\|f_{j} \mid L_{p}\right\|^{q}\right)^{1 / q}
$$

with the appropriate modification if $q=\infty$.

Definition 2.4 Let $N=\left(N_{j}\right)_{j \in \mathbb{N}_{0}}$ be an admissible sequence with $\underline{N}_{1}>1$ and $\varphi^{N}$ a system of functions as in Definition 2.3. Let $0<p, q \leq \infty$ and $\sigma=\left(\sigma_{j}\right)_{j \in \mathbb{N}_{0}}$ be an admissible sequence. Then the Besov space of generalized smoothness $B_{p, q}^{\sigma, N}$ is the set of all tempered distributions $f$ such that the quasi-norm

$$
\left\|f\left|B_{p, q}^{\sigma, N}\|:=\|\left(\sigma_{j} \varphi_{j}^{N}(D) f\right)_{j \in \mathbb{N}_{0}}\right| \ell_{q}\left(L_{p}\right)\right\|
$$

is finite.

Remark 2.5 Note that if $0<p<\infty$ then the Triebel-Lizorkin space of generalized smoothness $F_{p, q}^{\sigma, N}$ is defined in an analogous way, by interchanging the roles of the $L_{p}$ and $\ell_{q}$ quasi-norms.

If $\sigma=\left(2^{s j}\right)_{j \in \mathbb{N}_{0}}$, with $s$ a real number, and $N=\left(2^{j}\right)_{j \in \mathbb{N}_{0}}$ then the spaces $B_{p, q}^{\sigma, N}$ coincide with the usual Besov spaces $B_{p, q}^{s}$ and $F_{p, q}^{\sigma, N}$ coincide with the usual Triebel-Lizorkin spaces $F_{p, q}^{s}$. If we let $\sigma=\left(2^{s j} \Psi\left(2^{-j}\right)\right)_{j \in \mathbb{N}_{0}}$, where $\Psi$ is an admissible function in the sense of [6, 7] (see Example 2.1 (iii)), the corresponding Besov space coincide with the space $B_{p, q}^{(s, \Psi)}$ introduced by Edmunds and Triebel in [6, 7] and also considered by Moura in $[17,18]$. A similarly observation holds for the $F$-counterpart. 


\section{Characterization by approximation}

Let $N=\left(N_{j}\right)_{j \in \mathbb{N}_{0}}$ be an admissible sequence with $\underline{N}_{1}>1$ (recall (2.2)) and $k_{0} \in \mathbb{N}_{0}$ as in (2.5). Let $0<p \leq \infty$ and

$$
\mathcal{U}_{p}^{N}:=\left\{a=\left(a_{j}\right)_{j \in \mathbb{N}_{0}}: a_{j} \in \mathcal{S}^{\prime} \cap L_{p}, \operatorname{supp} \mathcal{F} a_{j} \subset\left\{y:|y| \leq N_{j+k_{0}}\right\}, j \in \mathbb{N}_{0}\right\} .
$$

Theorem 3.1 Let $\sigma=\left(\sigma_{j}\right)_{j \in \mathbb{N}_{0}}$ be an admissible sequence, $0<p, q \leq \infty$, and $N=\left(N_{j}\right)_{j \in \mathbb{N}_{0}}$ be an admissible sequence with $\underline{N}_{1}>1$, as above.

Suppose that $\underline{s}(\sigma) \bar{s}(N)^{-1}>n(1 / p-1)_{+}$. Then

$$
\begin{gathered}
B_{p, q}^{\sigma, N}=\left\{f \in \mathcal{S}^{\prime}: \exists a=\left(a_{j}\right)_{j \in \mathbb{N}_{0}} \in \mathcal{U}_{p}^{N} \text { such that } f=\lim _{k \rightarrow \infty} a_{k} \text { in } \mathcal{S}^{\prime}\right. \text { and } \\
\left.\left\|f\left|B_{p, q}^{\sigma, N}\left\|^{a}:=\right\| a_{0}\right| L_{p}\right\|+\left\|\left(\sigma_{k}\left(f-a_{k}\right)\right)_{k \in \mathbb{N}} \mid \ell_{q}\left(L_{p}\right)\right\|<\infty\right\} .
\end{gathered}
$$

Furthermore,

$$
\left\|f\left|B_{p, q}^{\sigma, N}\left\|^{X}:=\inf \right\| f\right| B_{p, q}^{\sigma, N}\right\|^{a},
$$

where the infimum is taken over all admissible systems $a \in \mathcal{U}_{p}^{N}$, is an equivalent quasi-norm in $B_{p, q}^{\sigma, N}$.

Proof. First of all let us remark that, taking into account the inequalities in (2.4), if $\underline{s}(\sigma) \bar{s}(N)^{-1}>\delta \geq 0$ then

$$
\left(\underline{\sigma}_{j}^{-1} \bar{N}_{j}^{\delta}\right)_{j \in \mathbb{N}} \in \ell_{u} \quad \text { for any } u \in(0, \infty]
$$

Step 1 . In this step we prove that $\left\|\cdot \mid B_{p, q}^{\sigma, N}\right\|^{X}$ can be estimated from above by $\left\|\cdot \mid B_{p, q}^{\sigma, N}\right\|$. Let $f \in B_{p, q}^{\sigma, N}$ and let $\left(\varphi_{j}^{N}\right)_{j=0}^{\infty}$ be a generalized partition of unity, according to Definition 2.3. Then

$$
a_{k}:=\sum_{j=0}^{k} \varphi_{j}^{N}(D) f \longrightarrow f \quad \text { in } \quad \mathcal{S}^{\prime} \quad \text { as } \quad k \longrightarrow \infty .
$$

Moreover, $a_{k} \in \mathcal{S}^{\prime} \cap L_{p}$ and $\operatorname{supp} \mathcal{F} a_{k} \subset\left\{y:|y| \leq N_{k+k_{0}}\right\}, k \in \mathbb{N}_{0}$. Hence, $a=\left(a_{k}\right)_{k \in \mathbb{N}_{0}} \in \mathcal{U}_{p}^{N}$. Let $\bar{p}:=\min (1, p)$ and put $\varphi_{l}^{N} \equiv 0$ if $-l \in \mathbb{N}$. Then

$$
\begin{aligned}
\sigma_{k}\left\|f-a_{k} \mid L_{p}\right\| & \leq \sigma_{k}\left(\sum_{j=k+1}^{\infty}\left\|\varphi_{j}^{N}(D) f \mid L_{p}\right\|^{\bar{p}}\right)^{1 / \bar{p}} \\
& \leq\left(\sum_{m=1}^{\infty} \underline{\sigma}_{m}^{-\bar{p}} \sigma_{k+m}^{\bar{p}}\left\|\varphi_{k+m}^{N}(D) f \mid L_{p}\right\|^{\bar{p}}\right)^{1 / \bar{p}} .
\end{aligned}
$$

If $0<q \leq \bar{p}$, from (3.1) and using the fact that $\left(\underline{\sigma}_{j}^{-1}\right)_{j \in \mathbb{N}_{0}} \in \ell_{q}$, we get

$$
\begin{aligned}
\left(\sum_{k=1}^{\infty} \sigma_{k}^{q}\left\|f-a_{k} \mid L_{p}\right\|^{q}\right)^{1 / q} & \leq\left(\sum_{k=1}^{\infty} \sum_{m=1}^{\infty} \underline{\sigma}_{m}^{-q} \sigma_{k+m}^{q}\left\|\varphi_{k+m}^{N}(D) f \mid L_{p}\right\|^{q}\right)^{1 / q} \\
& \leq\left\|\left(\underline{\sigma}_{j}^{-1}\right)_{j \in \mathbb{N}_{0}}\left|\ell_{q}\|\| f\right| B_{p, q}^{\sigma, N}\right\| \\
& \leq c\left\|f \mid B_{p, q}^{\sigma, N}\right\| .
\end{aligned}
$$

If $q>\bar{p}$, from (3.1) by using the triangle inequality with respect to $\ell_{q / \bar{p}}$ and $\left(\underline{\sigma}_{j}^{-1}\right)_{j \in \mathbb{N}_{0}} \in \ell_{\bar{p}}$, we obtain

$$
\left(\sum_{k=1}^{\infty} \sigma_{k}^{q}\left\|f-a_{k} \mid L_{p}\right\|^{q}\right)^{1 / q} \leq\left\{\sum_{m=1}^{\infty} \underline{\sigma}_{m}^{-\bar{p}}\left(\sum_{k=1}^{\infty} \sigma_{k+m}^{q}\left\|\varphi_{k+m}^{N}(D) f \mid L_{p}\right\|^{q}\right)^{\bar{p} / q}\right\}^{1 / \bar{p}} \leq
$$

www.mn-journal.com 


$$
\leq\left\|\left(\underline{\sigma}_{j}^{-1}\right)_{j \in \mathbb{N}_{0}}\left|\ell_{\bar{p}}\|\| f\right| B_{p, q}^{\sigma, N}\right\| \leq c\left\|f \mid B_{p, q}^{\sigma, N}\right\|
$$

(with the usual modification if $q=\infty$ ). Since we also have

$$
\left\|a_{0}\left|L_{p}\|=\| \varphi_{0}^{N}(D) f\right| L_{p}\right\| \leq c\left\|f \mid B_{p, q}^{\sigma, N}\right\|,
$$

then

$$
\left\|f\left|B_{p, q}^{\sigma, N}\left\|^{a} \leq c\right\| f\right| B_{p, q}^{\sigma, N}\right\|, \quad f \in B_{p, q}^{\sigma, N} .
$$

Step 2. Let $f \in \mathcal{S}^{\prime}$ and $a=\left(a_{k}\right)_{k \in \mathbb{N}_{0}} \in \mathcal{U}_{p}^{N}$ be such that

$$
f=\lim _{k \rightarrow \infty} a_{k} \text { in } \mathcal{S}^{\prime} \text { and }\left\|f \mid B_{p, q}^{\sigma, N}\right\|^{a}<\infty .
$$

Let $l_{0}=l_{0}(N) \in \mathbb{N}$ be such that $\underline{N}_{1}^{l_{0}}>\bar{N}_{1}$ and let $\mu_{0}, \mu \in \mathcal{S}$ be positive functions such that

$$
\mu_{0}(\xi)=1 \quad \text { if } \quad|\xi| \leq N_{1}, \quad \operatorname{supp} \mu_{0} \subset\left\{\xi \in \mathbb{R}^{n}:|\xi| \leq \underline{N}_{1} N_{1}\right\}
$$

and

$$
\mu(\xi)=1 \quad \text { if } \quad \bar{N}_{1}^{-1} \leq|\xi| \leq \bar{N}_{1}, \quad \operatorname{supp} \mu \subset\left\{\xi \in \mathbb{R}^{n}: \underline{N}_{1}^{-l_{0}} \leq|\xi| \leq \underline{N}_{1}^{l_{0}}\right\} .
$$

For each $j \in \mathbb{N}$ define $\mu_{j}(\xi):=\mu\left(N_{j}^{-1} \xi\right), \xi \in \mathbb{R}^{n}$. Putting $a_{l} \equiv 0$ if $-l \in \mathbb{N}$, we have

$$
\mu_{k}(D) f=\sum_{j=1-l_{0}-k_{0}}^{\infty} \mathcal{F}^{-1}\left[\mu_{k} \mathcal{F}\left(a_{k+j}-a_{k+j-1}\right)\right], \quad k \in \mathbb{N}_{0},
$$

and hence, with $\bar{p}$ as in step 1 , we get

$$
\left\|\mu_{k}(D) f \mid L_{p}\right\| \leq\left(\sum_{j=-1}^{\infty}\left\|\mathcal{F}^{-1}\left[\mu_{k} \mathcal{F}\left(a_{k+j}-a_{k+j-1}\right)\right] \mid L_{p}\right\|^{\bar{p}}\right)^{1 / \bar{p}}, \quad k \in \mathbb{N}_{0} .
$$

Recall that $\operatorname{supp} \mathcal{F}\left(a_{k+j}-a_{k+j-1}\right) \subset\left\{\xi:|\xi| \leq N_{k+j+k_{0}}\right\}$. By hypothesis $\underline{s}(\sigma) \bar{s}(N)^{-1}>n(1 / p-1)_{+}$, so that we can choose a real number $\lambda$ satisfying $\underline{s}(\sigma) \bar{s}(N)^{-1}>\lambda-n / 2>n(1 / p-1)_{+}$. Then the inequality (13) of $[19,1.5 .2$, p. 28$]$ yields

$$
\begin{aligned}
& \left\|\mathcal{F}^{-1}\left[\mu_{k} \mathcal{F}\left(a_{k+j}-a_{k+j-1}\right)\right] \mid L_{p}\right\| \\
& \quad \leq c\left\|\mu_{k}\left(N_{k+j+k_{0}} \cdot\right)\left|H_{2}^{\lambda}\|\| a_{k+j}-a_{k+j-1}\right| L_{p}\right\|, \quad k \in \mathbb{N}_{0},
\end{aligned}
$$

where $c$ is a positive constant independent of $k$ and $j$. Using [5, Proposition 1, p. 33] we obtain, for $k \in \mathbb{N}$,

$$
\begin{aligned}
\left\|\mu_{k}\left(N_{k+j+k_{0}} \cdot\right) \mid H_{2}^{\lambda}\right\| & =\left\|\mu\left(N_{k+j+k_{0}} N_{k}^{-1} \cdot\right) \mid H_{2}^{\lambda}\right\| \\
& \leq c_{1}\left(N_{k+j+k_{0}} N_{k}^{-1}\right)^{\lambda-\frac{n}{2}}\left\|\mu(a \cdot) \mid H_{2}^{\lambda}\right\| \leq c_{2} \bar{N}_{j}^{\lambda-\frac{n}{2}}
\end{aligned}
$$

where $a=a(N)$ and $c_{1}, c_{2}$ are positive constants independent of $j$ and $k$. In an analogous way, for $k=0$ we also have

$$
\left\|\mu_{0}\left(N_{j+k_{0}} \cdot\right) \mid H_{2}^{\lambda}\right\| \leq c \bar{N}_{j}^{\lambda-\frac{n}{2}} .
$$

Inserting (3.3)-(3.5) in (3.2), leads us to

$$
\left\|\mu_{k}(D) f \mid L_{p}\right\| \leq c\left(\sum_{j=1-l_{0}-k_{0}}^{\infty} \bar{N}_{j}^{\left(\lambda-\frac{n}{2}\right) \bar{p}}\left\|a_{k+j}-a_{k+j-1} \mid L_{p}\right\|^{\bar{p}}\right)^{1 / \bar{p}}
$$


(with the understanding that $\bar{N}_{j} \equiv 1$ if $-j \in \mathbb{N}$ ). If $0<q \leq \bar{p}$, it follows

$$
\begin{aligned}
\sum_{k=0}^{\infty} \sigma_{k}^{q}\left\|\mu_{k}(D) f \mid L_{p}\right\|^{q} & \leq c_{1} \sum_{k=0}^{\infty} \sigma_{k}^{q} \sum_{j=1-l_{0}-k_{0}}^{\infty} \bar{N}_{j}^{\left(\lambda-\frac{n}{2}\right) q}\left\|a_{k+j}-a_{k+j-1} \mid L_{p}\right\|^{q} \\
& \leq c_{2}\left\|\left(\bar{N}_{j}^{\left(\lambda-\frac{n}{2}\right)} \underline{\sigma}_{j}^{-1}\right)_{j \in \mathbb{N}_{0}}\left|\ell_{q}\left\|^{q} \sum_{m=0}^{\infty} \sigma_{m}^{q}\right\| a_{m}-a_{m-1}\right| L_{p}\right\|^{q} \\
& \leq c_{3}\left\|a_{0}\left|L_{p}\left\|+c_{4} \sum_{m=0}^{\infty} \sigma_{m}^{q}\right\| f-a_{m}\right| L_{p}\right\|^{q}
\end{aligned}
$$

where we have used the fact that $\left(\bar{N}_{j}^{\left(\lambda-\frac{n}{2}\right)}{\underline{\sigma_{j}}}^{-1}\right)_{j \in \mathbb{N}_{0}} \in \ell_{q}$, which is a consequence of the choice of $\lambda$. On the other hand, if $q>\bar{p}$, reasoning as in step 1, we obtain

$$
\begin{aligned}
\sum_{k=0}^{\infty} \sigma_{k}^{q}\left\|\mu_{k}(D) f \mid L_{p}\right\|^{q} & \leq c_{1}\left\|\left(\bar{N}_{j}^{\left(\lambda-\frac{n}{2}\right)} \underline{\sigma}_{j}^{-1}\right)_{j \in \mathbb{N}_{0}}\left|\ell_{\bar{p}}\left\|^{q} \sum_{m=0}^{\infty} \sigma_{m}^{q}\right\| a_{m}-a_{m-1}\right| L_{p}\right\|^{q} \\
& \leq c_{2}\left\|a_{0}\left|L_{p}\left\|+c_{3} \sum_{m=0}^{\infty} \sigma_{m}^{q}\right\| f-a_{m}\right| L_{p}\right\|^{q}
\end{aligned}
$$

(with the usual modification if $q=\infty$ ). Using now the [8, Theorem 4.2.2], by (3.6) and (3.7) we get, for any $q \in(0, \infty]$

$$
\left\|f\left|B_{p, q}^{\sigma, N}\|\leq c\| f\right| B_{p, q}^{\sigma, N}\right\|^{a}, \quad f \in B_{p, q}^{\sigma, N}
$$

where $c$ is a positive constant independent of the chosen system $a=\left(a_{k}\right)_{k \in \mathbb{N}_{0}}$. Therefore,

$$
\left\|f\left|B_{p, q}^{\sigma, N}\|\leq c\| f\right| B_{p, q}^{\sigma, N}\right\|^{X}, \quad f \in B_{p, q}^{\sigma, N} .
$$

Remark 3.2 For $N=\left(2^{j}\right)_{j \in \mathbb{N}_{0}}$ and $\sigma=\left(2^{s j}\right)_{j \in \mathbb{N}_{0}}$ or $\sigma=\left(2^{s j} \Phi\left(2^{-j}\right)\right)_{j \in \mathbb{N}_{0}}$ (cf. Examples 2.1 (i) and (ii)), the above theorem coincide with [19, Theorem 2.5.3 (i)] and [11, Theorem 2.4], respectively.

A characterization as above is valid for the space $F_{p, q}^{\sigma, N}$, replacing the condition $\underline{s}(\sigma) \bar{s}(N)^{-1}>n(1 / p-1)_{+}$ by $\underline{s}(\sigma) \bar{s}(N)^{-1}>n(1 / \min (p, q)-1)_{+}$and interchanging the roles of the $L_{p}$ and $\ell_{q}$ quasi-norms.

\section{Characterization by differences}

Next we recall the definition of differences of functions. If $f$ is an arbitrary function on $\mathbb{R}^{n}, h \in \mathbb{R}^{n}$ and $k \in \mathbb{N}$, then

$$
\left(\Delta_{h}^{k} f\right)(x):=\sum_{j=0}^{k}\left(\begin{array}{l}
k \\
j
\end{array}\right)(-1)^{k-j} f(x+j h), \quad x \in \mathbb{R}^{n} .
$$

Note that $\Delta_{h}^{k}$ can also be defined iteratively via

$$
\left(\Delta_{h}^{1} f\right)(x)=f(x+h)-f(x) \quad \text { and } \quad\left(\Delta_{h}^{k+1} f\right)(x)=\Delta_{h}^{1}\left(\Delta_{h}^{k} f\right)(x), \quad k \in \mathbb{N} .
$$

Furthermore, the $k$-th modulus of smoothness of a function $f \in L_{p}, 1 \leq p \leq \infty, k \in \mathbb{N}$, is defined by

$$
\omega_{k}(f, t)_{p}=\sup _{|h| \leq t}\left\|\Delta_{h}^{k} f \mid L_{p}\right\|, \quad t>0
$$


Theorem 4.1 Let $0<p, q \leq \infty, \sigma=\left(\sigma_{j}\right)_{j \in \mathbb{N}_{0}}$ and $N=\left(N_{j}\right)_{j \in \mathbb{N}_{0}}$ be admissible sequences, the latter satisfying $\underline{N}_{1}>1$, such that $\underline{s}(\sigma) \bar{s}(N)^{-1}>n(1 / p-1)_{+}$. Let $M \in \mathbb{N}, M>\bar{s}(\sigma) \underline{s}(N)^{-1}$. Then

$$
B_{p, q}^{\sigma, N}=\left\{f \in L_{\max (1, p)}:\left\|f\left|B_{p, q}^{\sigma, N}\left\|^{(M)}:=\right\| f\right| L_{p}\right\|+\left(\sum_{j=0}^{\infty} \sigma_{j}^{q} \omega_{M}\left(f, N_{j}^{-1}\right)_{p}^{q}\right)^{1 / q}<\infty\right\}
$$

(with the usual modification if $q=\infty$ ) in the sense of equivalent quasi-norms.

Remark 4.2 It can be easily verified that, for any $a \in(0,+\infty)$,

$$
\left\|f\left|B_{p, q}^{\sigma, N}\left\|^{(M)} \sim\right\| f\right| L_{p}\right\|+\left(\int_{0}^{a}\left(\Lambda\left(t^{-1}\right) \omega_{M}(f, t)_{p}\right)^{q} \frac{\mathrm{d} t}{t}\right)^{1 / q}
$$

where $\Lambda:(0, \infty) \rightarrow(0, \infty)$ is, according to [3, Def. 2.2.1], an admissible function such that $\Lambda(z) \sim \Lambda\left(N_{j}\right)=$ $\sigma_{j}, z \in\left[N_{j}, N_{j+1}\right], j \in \mathbb{N}_{0}$.

Pro of. Step 1. In this step we shall prove that there exists a positive constant $c$ such that

$$
\left\|f\left|B_{p, q}^{\sigma, N}\left\|^{(M)} \leq c\right\| f\right| B_{p, q}^{\sigma, N}\right\|
$$

holds for all $f \in B_{p, q}^{\sigma, N}$. If $f \in B_{p, q}^{\sigma, N}$ with $\underline{s}(\sigma) \bar{s}(N)^{-1}>n(1 / p-1)_{+}$, then $f \in L_{\max (1, p)}$ and

$$
\left\|f\left|L_{p}\|\leq c\| f\right| B_{p q}^{\sigma, N}\right\|
$$

for some positive constant $c$ which does not depend on $f$. This is a consequence of [3, Corollary 3.18], as $\left(\sigma_{j}^{-1} N_{j}^{n\left(\frac{1}{p}-1\right)_{+}}\right) \in \ell_{q^{\prime}}$ in this case.

With $\left(\varphi_{j}^{N}\right)_{j \in \mathbb{N}_{o}}$ being a generalized partition of unity according to Definition 2.3 we have, with convergence in $L_{p}$,

$$
f=\sum_{j=0}^{\infty} \varphi_{j}(D) f
$$

Let $a>n / p$. If $k \in \mathbb{N}_{0}$ then for each $\varrho \in \mathbb{R}^{n}$ with $|\varrho| \leq N_{k}^{-1}$ and $j \in\{0, \ldots, k\}$ we have

$$
\begin{aligned}
\left|\Delta_{\varrho}^{M}\left(\varphi_{j}^{N}(D) f\right)(x)\right|= & \left|\sum_{\ell=0}^{M}\left(\begin{array}{c}
M \\
\ell
\end{array}\right)(-1)^{M-\ell}\left(\varphi_{j}^{N}(D) f\right)(x+\ell \varrho)\right| \\
\leq & \left|\sum_{\ell=0}^{M}\left(\begin{array}{c}
M \\
\ell
\end{array}\right)(-1)^{M-\ell} \sum_{|\beta| \leq M-1} \frac{D^{\beta}\left(\varphi_{j}^{N}(D) f\right)(x)}{\beta !}(\ell \varrho)^{\beta}\right| \\
& +\left|\sum_{\ell=0}^{M}\left(\begin{array}{c}
M \\
\ell
\end{array}\right)(-1)^{M-\ell} \sum_{|\alpha|=M} \frac{D^{\alpha}\left(\varphi_{j}^{N}(D) f\right)\left(x_{j, \varrho}\right)}{\alpha !}(\ell \varrho)^{\alpha}\right| \\
\leq & c_{1} N_{k}^{-M} \sup _{|y-x| \leq N_{k}^{-1}} \sum_{|\alpha|=M}\left|D^{\alpha}\left(\varphi_{j}^{N}(D) f\right)(y)\right| \\
\leq & c_{2} N_{j}^{M} N_{k}^{-M} \sup _{|y-x| \leq N_{k}^{-1}} \sup _{z \in \mathbb{R}^{n}} \frac{\left|\left(\varphi_{j}^{N}(D) f\right)(y-z)\right|}{1+\left|N_{j+1} z\right|^{a}} \\
\leq & c_{3} \underline{N}_{k-j}^{-M}\left(\varphi_{j}^{N *} f\right)_{a}(x),
\end{aligned}
$$

where $\left(\varphi_{j}^{N *} f\right)_{a}$ denotes the Peetre's maximal functions. Besides Taylor's expansion we have used the fact that 
$\sum_{\ell=0}^{M}\left(\begin{array}{c}M \\ \ell\end{array}\right)(-1)^{M-\ell} \ell^{m}=0$ for any $m \in \mathbb{N}_{0}$ with $m<M$, and [19, Theorem 1.3.1,p. 16] for the third inequality. Hence, for $j \in \mathbb{N}_{0}$,

$$
\sup _{|\varrho| \leq N_{k}^{-1}}\left\|\Delta_{\varrho}^{M}\left(\varphi_{j}^{N}(D) f\right)\left|L_{p}\left\|\leq c \min \left(1, \underline{N}_{k-j}^{-M}\right)\right\|\left(\varphi_{j}^{N *} f\right)_{a}\right| L_{p}\right\|,
$$

where $c>0$ is a constant independent of $f$.

Suppose that $0<p \leq 1$ (otherwise, similarly to the proof of the previous theorem, one has to modify the calculations below in an obvious way), then, by (4.3) and (4.5), we get

$$
\begin{aligned}
\sup _{|\varrho| \leq N_{k}^{-1}}\left\|\Delta_{\varrho}^{M} f \mid L_{p}\right\|^{p} & \leq \sum_{j=0}^{\infty} \sup _{|\varrho| \leq N_{k}^{-1}}\left\|\Delta_{\varrho}^{M}\left(\varphi_{j}^{M}(D) f\right) \mid L_{p}\right\|^{p} \\
& \leq c \sum_{j=0}^{k} \underline{N}_{k-j}^{-M p}\left\|\left(\varphi_{j}^{N *} f\right)_{a}\left|L_{p}\left\|^{p}+c \sum_{j=k+1}^{\infty}\right\|\left(\varphi_{j}^{N *} f\right)_{a}\right| L_{p}\right\|^{p} .
\end{aligned}
$$

With $\Lambda$ as in Remark 4.2, it follows

$$
\begin{aligned}
\left\|f \mid B_{p, q}^{\sigma, N}\right\|^{(M) q} \leq & c_{1}\left\|f \mid L_{p}\right\|^{q}+c_{1} \int_{0}^{N_{0}^{-1}}\left(\Lambda\left(t^{-1}\right) \omega_{M}(f, t)_{p}\right)^{q} \frac{\mathrm{d} t}{t} \\
\leq & c_{1}\left\|f \mid L_{p}\right\|^{q}+c_{1} \sum_{k=0}^{\infty} \int_{N_{k+1}^{-1}}^{N_{k}^{-1}}\left(\Lambda\left(t^{-1}\right) \omega_{M}(f, t)_{p}\right)^{q} \frac{\mathrm{d} t}{t} \\
\leq & c_{1}\left\|f \mid L_{p}\right\|^{q}+c_{2} \sum_{k=0}^{\infty} \sigma_{k}^{q} \omega_{M}\left(f, N_{k}^{-1}\right)_{p}^{q} \\
\leq & c_{1}\left\|f \mid L_{p}\right\|^{q}+c_{3} \sum_{k=0}^{\infty} \sigma_{k}^{q}\left(\sum_{j=0}^{k} \underline{N}_{k-j}^{-M p}\left\|\left(\varphi_{j}^{N *} f\right)_{a} \mid L_{p}\right\|^{p}\right)^{q / p} \\
& +c_{3} \sum_{k=0}^{\infty} \sigma_{k}^{q}\left(\sum_{j=k+1}^{\infty}\left\|\left(\varphi_{j}^{N *} f\right)_{a} \mid L_{p}\right\|^{p}\right)^{q / p}
\end{aligned}
$$

(with the usual modification if $q=\infty$ ). By hypothesis $M>\bar{s}(\sigma) \underline{s}(N)^{-1}$, so that (2.4) entails $\left(\bar{\sigma}_{j} \underline{N}_{j}^{-M}\right)_{j \in \mathbb{N}_{0}} \in$ $\ell_{u}$, for any $u \in(0, \infty]$. Then we obtain

$$
\begin{aligned}
& \sum_{k=0}^{\infty} \sigma_{k}^{q}\left(\sum_{j=0}^{k} \underline{N}_{k-j}^{-M p}\left\|\left(\varphi_{j}^{N *} f\right)_{a} \mid L_{p}\right\|^{p}\right)^{q / p} \\
& \quad \leq c\left\|\left(\bar{\sigma}_{j} \underline{N}_{j}^{-M}\right)_{j \in \mathbb{N}_{0}}\left|\ell_{\min (1, p, q)}\left\|^{q} \sum_{j=0}^{\infty} \sigma_{j}^{q}\right\|\left(\varphi_{j}^{N *} f\right)_{a}\right| L_{p}\right\|^{q}
\end{aligned}
$$

and also

$$
\begin{aligned}
& \sum_{k=0}^{\infty} \sigma_{k}^{q}\left(\sum_{j=k+1}^{\infty}\left\|\left(\varphi_{j}^{N *} f\right)_{a} \mid L_{p}\right\|^{p}\right)^{q / p} \\
& \quad \leq c\left\|\left(\underline{\sigma}_{j}^{-1}\right)_{j \in \mathbb{N}_{0}}\left|\ell_{\min (1, p, q)}\left\|^{q} \sum_{j=0}^{\infty} \sigma_{j}^{q}\right\|\left(\varphi_{j}^{N *} f\right)_{a}\right| L_{p}\right\|^{q} .
\end{aligned}
$$

We insert (4.8) and (4.9) in (4.7). Then (4.1) is a consequence of [3, Theorem 4.3.4] and (4.2).

Step 2. Let $f \in B_{p, q}^{\sigma, N}$. By Theorem 3.1,

$$
\left\|f\left|B_{p, q}^{\sigma, N}\|\leq c\| f\right| L_{p}\right\|+c\left(\sum_{j=1}^{\infty} \sigma_{j}^{q} E_{p}\left(N_{j+k_{0}}, f\right)^{q}\right)^{1 / q}
$$


for some positive constant $c$ and with

$$
E_{p}(b, f):=\inf \left\|f-g \mid L_{p}\right\|
$$

where the infimum is taken over all $g \in \mathcal{S}^{\prime} \cap L_{p}$ such that $\operatorname{supp} \widehat{g} \subset\{y:|y| \leq b\}$. As there is a positive constant $c$ (which depends on $p$ and $k$ ) such that

$$
E_{p}(b, f) \leq c \sup _{|h| \leq b^{-1}}\left\|\Delta_{h}^{M} f \mid L_{p}\right\|
$$

for all $b \geq 1$ and $f \in L_{p}$ (cf. [19, Prop. 2.5.12, p. 109]) from (4.10) we get

$$
\left\|f\left|B_{p, q}^{\sigma, N}\left\|\leq c_{1}\right\| f\right| L_{p}\right\|+c_{2}\left(\sum_{j=1}^{\infty} \sigma_{j}^{q} \omega^{M}\left(f, N_{j+k_{0}}^{-1}\right)_{p}^{q}\right)^{1 / q} \leq c_{3}\left\|f \mid B_{p, q}^{\sigma, N}\right\|^{(M)}
$$

which completes the proof.

Remark 4.3 For $N=\left(2^{j}\right)_{j \in \mathbb{N}_{0}}$ and $\sigma=\left(2^{s j}\right)_{j \in \mathbb{N}_{0}}$ or $\sigma=\left(2^{s j} \Phi\left(2^{-j}\right)\right)_{j \in \mathbb{N}_{0}}$ (cf. Examples 2.1 (i) and (ii)), the above theorem is covered by [19, Theorem 2.5.12] and [11, Theorem 2.5], respectively. For $1<p<\infty$, $1<q \leq \infty$ this type of characterization has been obtained by Kalyabin in [14].

Acknowledgements The author is grateful to Professor H.-G. Leopold for valuable remarks concerning the subject. This work was partially supported by Centro de Matemática da Universidade de Coimbra and by a project under the agreement GRICES/DAAD.

\section{References}

[1] N. H. Bingham, C. M. Goldie, and J. L. Teugels, Regular Variation (Cambridge University Press, Cambridge, 1987).

[2] M. Bricchi, Tailored Function Spaces and $h$-sets, Ph.D. thesis, University of Jena (2002).

[3] A. M. Caetano and W. Farkas, Local growth envelopes of Besov spaces of generalized smoothness, Z. Anal. Anwend. 25, No. 3, 265-298 (2006).

[4] F. Cobos and D. Fernandez, Hardy-Sobolev spaces and Besov spaces with a function parameter, in: Lecture Notes in Mathematics Vol. 1302 (Springer, Berlin, 1988), pp. 158-170.

[5] D. E. Edmunds and H. Triebel, Function Spaces, Entropy Numbers and Differential Operators (Cambridge University Press, Cambridge, 1996).

[6] D. E. Edmunds and H. Triebel, Spectral theory for isotropic fractal drums, C. R. Acad. Sci. Paris Sér. I Math. 326, 1269-1274 (1998).

[7] D. E. Edmunds and H. Triebel, Eigenfrequencies of isotropic fractal drums, Oper. Theory Adv. Appl. 110, 81-102 (1999).

[8] W. Farkas and H.-G. Leopold, Characterizations of function spaces of generalized smoothness, Ann. Mat. Pura Appl., IV. Ser., to appear.

[9] M. L. Goldman, A description of the traces of some function spaces, Tr. Mat. Inst. Steklova 150, 99-127 (1979) (in Russian).

[10] M. L. Gold'man, A method of coverings for describing general spaces of Besov type, Tr. Mat. Inst. Steklova 156, 47-81 (1980) (in Russian).

[11] D. D. Haroske and S. D. Moura, Continuity envelopes of spaces of generalized smoothness, entropy and approximation numbers, J. Approx. Theory 128, No. 2, 151-174 (2004).

[12] G. A. Kalyabin and P. I. Lizorkin, Spaces of generalized smoothness, Math. Nachr. 133, 7-32 (1987).

[13] G. A. Kalyabin, Characterization of spaces of generalized Liouville differentiation, Mat. Sb. (N.S.) 104, $42-48$ (1977) (in Russian).

[14] G. A. Kalyabin, Description of functions from classes of Besov-Lizorkin-Triebel type, Tr. Mat. Inst. Steklova 156, 82-109 (1980) (in Russian).

[15] H.-G. Leopold, Embeddings and entropy numbers in Besov spaces of generalized smoothness, in: Function Spaces: The Fifth Conference, Lecture Notes in Pure and Applied Mathematics Vol. 213 (Marcel Dekker, New York, 2000), pp. 323-336.

[16] C. Merucci, Applications of interpolation with a function parameter to Lorentz, Sobolev and Besov spaces, in: Lecture Notes in Mathematics Vol. 1070 (Springer, Berlin, 1984), pp. 183-201. 
[17] S. Moura, Function spaces of generalized smoothness, Diss. Math. 398, 1-88 (2001).

[18] S. Moura, Function Spaces of Generalized Smoothness, Entropy Numbers, Applications, Ph.D. thesis, University of Coimbra (2001).

[19] H. Triebel, Theory of Function Spaces (Birkhäuser, Basel, 1983). 